\title{
ACCESS TO OPPORTUNITY: THE DUKE-EAST CAROLINA PARTNERSHIPS FOR TRAINING PROGRAM
}

\author{
Mary T. Champagne RN, Ph.D. \\ Duke University School of Nursing \\ Box 3322 Medical Center \\ Durham, North Carolina 27710 \\ Telephone: 919-684-3786 ext. 225 \\ Fax: 919-681-8899 \\ Email: champ001@mc.duke.edu
}

Donna Hewitt, RN, MN

Box 3322 Duke University Medical Center

Durham, North Carolina 27710

Telephone: 919-684-3786 ext. 256

Fax: 919-681-8899

Email: hewit001@mc.duke.edu

Nancy Short, RN, MBA

2318 Thunder Road

Durham, N.C. 27712

Telephone: 919-383-5166

Fax: 919-382-1978

Email: nshort@nc.rr.com

Susan Pietrangelo-Brown, RN, MSN, FNP

Email: smbrown@nc.rr.com

Susan Epstein, MPA

Telephone: 919-681-3187

Email: epste003@mc.duke.edu

Margaret Bowers, RN, MSN, FNP

Telephone: 919-681-3244

Email: Bower005@mc.duke.edu 


\begin{abstract}
Duke University and East Carolina University through their Schools of Nursing and Physician Assistant Programs are in the third year of the Partnerships for Training Program (PFT). The goal of PFT is to prepare primary care providers to increase access to care in medically underserved and health professional shortage areas. This paper discusses how an asynchronous online mode was used to bring the universities and all of their resources to rural students in North Carolina. In addition, preparing students to access the online university and preparing faculty to teach using this new medium are discussed.
\end{abstract}

\title{
KEYWORDS
}

Collaborative education, Health care, Access, Partnerships

\section{DEDICATION}

This paper is dedicated to the students in the PFT classes of 2001 and 2003, who pioneered earning their master's degree's online, with a spirit of intellectual inquiry and hard work, a sense of humor and collegiality, and a deep caring for the communities in which they live.

\section{OVERVIEW OF THE PROGRAM}

In 1995, Duke University and East Carolina University responded to a call from the Robert Wood Johnson Foundation to develop a program for the collaborative education of Physician Assistants, Family Nurse Practitioners and Nurse Midwives. A key goal of "Partnerships for Training" (PFT) was to prepare primary care providers to increase access to care in medically underserved and health professional shortage areas (MUAs and HPSAs). In 1997, following two years of planning, the Robert Wood Johnson Foundation and the Duke Endowment funded implementation of our program. For both universities, the funding provided a golden opportunity to improve access to health care in our largely rural state, to explore collaborative models of education, and to test a new way of delivering education.

We initially targeted a 32-county area in eastern North Carolina. The population of the region is predominantly rural, poor, and underserved. Only four counties are classified as metropolitan areas and only a fifth of the region's people live in towns with over 10,000 people. Compared with North Carolina as a whole, the region has more low-income people, a larger minority population, a higher unemployment rate, and fewer college graduates [1]. It is not surprising, then, that most counties in the region have more than their share of health problems. Heart disease mortality rates are among the highest in the state, and the rate in every county exceeds the U.S. Public Health Service's objective of no more than 100 deaths from heart disease per 100,000. The statistics are comparable for cancer, diabetes, and death rates for stroke and chronic obstructive pulmonary disease. Infant mortality rates are among the worst in the nation [2]. Twenty-five of the region's 32 counties are designated Health Professional Shortage Areas and all are Medically Underserved Areas. The ratio of people per primary care provider in the region is significantly higher than that for North Carolina as a whole and double the ratio for the United States [3]. Clearly there is a need to educate primary care providers who are committed to these rural communities. Our PFT program is based on the premise that students recruited from this rural region and educated in their communities using innovative asynchronous learning methods will stay and practice in those communities following graduation. 
The Duke-East Carolina Partnership for Training Program is complex. Imagine, if you can, the very beginning of the partnership. Faculty from two very different universities, one public and one private, each with dearly held cultures, were called upon to plan a joint program. Faculty from family nurse practitioner, physician assistant, and nurse midwifery programs were asked to develop interdisciplinary courses. All were asked to accept more students, recruit non-traditional students from underserved areas, and develop new rural clinical training sites. They were tasked with coordinating the admission of students among programs and giving admission preference to qualified applicants who are committed to their rural communities. And finally, they were asked to deliver the program using web-based asynchronous learning methods - at a time when "dot.com" was not in the popular lexicon.

Community partners helped us develop our program. Our PFT partners included four regional Area Health Education Centers (AHECs) (Southern Regional, Eastern, Area L, and Costal AHEC), the statewide North Carolina AHEC, five rural hospitals (Carteret General, Sampson Regional, Southeastern Regional, Martin General, and Chowan General) three professional associations (the North Carolina Nurses' Association, the North Carolina Academy of Physician Assistants, and the North Carolina Chapter of the American College of Nurse Midwives), two state universities (one historically Black and one historically Native American), the State Medical Society Foundation and the State Office of Rural Health. These partners not only provided expertise, but they also kept our eye on the goal - educational opportunities for rural health care students and greater access to care for rural residents. Surely mere curricular difficulties could be overcome when the stakes were so high!

We are now entering the third year of our program. Eighty-six students are enrolled in the PFT program, either in the class of 2001 or the class of 2003. All reside in MUAs and $60 \%$ live in HPSAs. Twenty percent are under-represented minorities. Coursework is delivered asynchronously using a Lotus Notes/Domino platform and, more recently, Blackboard TM. Students study part-time, usually taking two courses a semester, three semesters a year. Over the last 3 years, over thirty faculty from our two universities have collectively taught 35 courses. Student performance parallels that of our on-campus students, and faculty enjoy using a new pedagogy and educational delivery system.

Our partnership and how we got from "there to here" is complex. While there are many lessons to be shared from our experience, our approach to "access" using an asynchronous learning delivery system is perhaps the most fundamental.

\section{WELCOME TO THE FOYER?}

Early in our planning, discussions centered on how we could bring quality education to our PFT students. There was concern that students would not have real access to the resources of the university but would only have the ability to enter the "foyer". At the heart of the matter, was our notion of the university and its value. We were well aware that we were in the business of education, not campus life. Yet, we valued the academic community as a model for educating and socializing future health care providers. The art and science of care require both critical thinking and connectedness. Our program, including online asynchronous delivery, focuses on what we see as key aspects of an academic community: shared pursuit of knowledge and intellectual growth, dynamic interaction among students and among students and faculty, belonging and shared values, and the development of collegial relationships. Bringing the university and all of its resources to students was therefore critical in creating access to our academic community. Providing students with the skills necessary to access the "university" and focus on learning 
rather than on technology is also critical. And finally, providing faculty with access to the support needed to effectively teach, work with students, and focus on student learning while using a new medium is a third critical component.

\section{BRINGING THE UNIVERSITY TO THE STUDENTS}

Frequent travel to campus is impossible for our students. They live at distant sites, most work full time, and many have families with young children. Thus, we knew that our university had to be available to students in an asynchronous mode, providing early morning, late evening and weekend access. During the planning phase of our program, few if any commercial Internet courseware packages were available. To ensure the simultaneous development of essential educational and technological components, we sought the services of an instructional technology consultant from the Institute of Academic Technology, which at the time was a research branch of IBM. The consultant and faculty worked together to develop our program. Faculty focused on essential components of the university and coursework; the consultant developed a platform to achieve the educational aims. The delivery system is housed on the School of Nursing server and uses a Lotus Notes/Domino platform. A single portal or homepage provides online entry for all students and faculty.

The homepage has a navigational bar that brings the university resources to the student. The online library provides access to the Duke and East Carolina libraries and to our partner AHEC libraries. There are also direct links to OVID and other databases, online journals, the online card catalog and government sites. Students can order an article or book directly from the online library using a built in document delivery service. The online library also has a "miscellaneous resource" section: students can link to clinical/health resources where they can access online learning modules such as breath sounds or EKG interpretation, and they can link to numerous other resources including a "fun site" which includes everything from airline tickets to the Duke News Service.

Other university resources available from the homepage include online course registration, financial aid and student services, an announcement or "new news" site, research opportunities, and usual materials such as the student handbook, the bulletin and the academic calendar. Helpline information and contacts and tutorials on computer-based instruction are also available.

To promote interaction, faculty, staff and students can be accessed from a navigational bar on the homepage via email. A general "student lounge," which only students can enter, allows our PFT students to have "hallway" or "lunch time conversations" or to discuss whatever students discuss without faculty around. Likewise, PFT faculty, who are housed in different universities and different departments, have a "faculty forum" which only faculty can enter.

Finally, the homepage provides access to the online courses. Health professional students need to acquire a broad knowledge base, they must learn to problem solve in complex clinical situations, and they must learn to consult with others, seek information when they are uncertain, and continue to learn as the evidence base for providing care expands. As we worked with our consultant to develop online course templates, we gave thought to each of these educational needs.

Faculty have the academic freedom to structure their online course as they choose. All of our courses, however, have a similar look and use common navigational and educational components. In general, the course contains usual information such as the syllabus and calendar, a page for 
each "class" of the semester that organizes the week's learning activities, and an academic forum. Students can work ahead; however, assignments are due are at set times and discussion forums are ongoing, which necessitates keeping up with course work. Class lectures are delivered using audio/video RealPlayer, notes from the lectures are printed with a mouse click, and readings are available through usual assigned texts and online electronic journals. Online quizzes that provide instant feedback are used with each class as pre or post tests to help students evaluate their own understanding of the content and their ability to apply the content in clinical situations. On the class page, faculty make frequent use of links to other web sites which have additional relevant material or learning activities.

The academic forum is part of each course and is used by faculty and students on a weekly basis for work that involves problem solving such as case studies, policy development, and ethical dilemmas, or to discuss implications of recent research for current practice. The forum presents excellent asynchronous opportunities for critical thinking, consultation among faculty and students, and learning when to seek additional information. The forum has two components: a general academic forum for all students and a "team section." Faculty use the team section to group students into manageable numbers for rigorous threaded discussions. For example, a course with 32 students might have a team forum with four teams of 8 students each. Faculty and students use the general forum to post discussion questions for the whole class.

Finally, our online courses offer opportunities to test and complete course evaluations online. Most tests are given on the honor system and are timed so that after a pre-set period the testing session ends and the student's work is sent to the faculty. Some faculty, however, prefer proctored exams. These are given online in our AHEC libraries, which are closer to the student's homes than campus.

\section{ENSURING STUDENT ACCESS TO THE ONLINE UNIVERSITY}

We were concerned that our students might have technical difficulties in accessing our online program. Stories of "crashed" systems, students who were computer "illiterate," and woes with inadequate hardware and software were heard daily. In addition, the mean age of our students was 35 , nearly all had completed college prior to the "computer revolution," and most had lived in their rural communities for an extended period. It was likely that most had little, if any, experience with web-based materials. Our aim was to eliminate potential technological barriers so that students could focus on their work. Therefore, prior to the first course offering, staff and faculty implemented a multi-step approach to prepare students for successful online learning. Along with the offer of admission to the program, students were given a list of computer hardware specifications and instructed to obtain an Internet Service Provider. During an oncampus orientation to the program, students brought their CPU unit to the School of Nursing, where a tech team ran diagnostics on each CPU and loaded all the software and plug-ins needed to access course materials. Using a custom-made CD to load the software, each CPU was serviced in 20 to 30 minutes. Students picked up their units the next day, along with a summary of what had been done to the unit and any problems detected. A few students had machines with inadequate memory or programs that needed to be deleted. Although we expected this approach to ensure that students would have the equipment necessary to access learning activities delivered online, we took additional steps to deal with possible computer failures. Our tech team traveled to our rural hospital and regional AHEC partners and configured computers stationed there with all necessary software and plug-ins. Finally, we purchased five back-up laptops that students could borrow if their computer failed. 
Students were asked to complete a Computer Skills Assessment Tool (CSAT), adapted with permission from a tool developed by the Massachusetts Institute of Technology. Using a Likert scale format, the CSAT assessed students' level of comfort with the computer in several domains, including operating systems, graphical user interface and windows tasks, common applications and email, file management, and use of the web. Although it later became clear that students tended to overestimate their computer skills, the majority reported significant learning needs related to file management, attaching files and downloading web materials. Student scores on the CSAT were used to give individual feedback to students (including recommending basic computer courses) and to plan a computer orientation session for all students on-campus prior to the beginning of coursework.

A student manual, a computer class, and a Help-Line were used to help students gain skill in accessing course material and dealing with problems. Faculty developed the student manual, which included both technical information as well as usual information given to any new student regarding student services and so forth. Topics included Your Computer, The Online Library, Help When You Need It, Your Mentor, Meet Your Classmates and The Faculty, Course Schedules, and Resources in Your Region. Students were given a hard copy of the manual, which could also be accessed online. We found that the class of 2001 never read the manual until they had a problem; consequently, we have required the class of 2003 to complete tutorials in the manual and send them to their faculty using email. During orientation, a hands-on computer class was used to teach students the fundamentals of accessing the web-based courses and gain fundamental skill in online learning. Finally, a toll-free phone line with evening and weekend hours was established to connect students experiencing online problems to technology experts who had a listing of all students and their computer specifications.

Being prepared has paid off. Technical problems have been few. Three of our students have had "drive failure" and used our backup systems. Some students have been unexpectedly bumped off the Internet. Students found this "catastrophic" if they were taking a "secure timed" exam. We have explored this problem and now recommend ISPs that provide more stable service, and we look forward to the day when rural areas will have DSLs. Our Help-Line is used infrequently. Most often, questions surface at the beginning of a semester when we enhance learning activities using new or updated software and students forget to install it. Nevertheless, the Help-Line is available when needed and when used, it does prevent cyber-frustration. There is a student learning curve to being "at home" online, but early positive experiences beget success. For our students, the computer has become a resource that helps them complete a rigorous course of study and an ally in life-long learning.

\section{FACULTY ACCESS TO TEACHING ONLINE}

Core faculty were involved in the development of our online PFT program from its inception. For the most part, they were faculty who were intrigued by innovation and had a keen desire to develop expertise in a new educational medium and test a new delivery system. They identified the advantages and limitations of our online asynchronous program and were instrumental in the development of methods to enhance the program - both technologically and pedagogically. As our program and online capabilities developed, we sought to increase the number of faculty prepared to teach online. Initially, we scheduled learning sessions for all faculty, providing a hands-on opportunity to put a class online. While this served to acquaint faculty with the idea of online asynchronous learning, many faculty remained uninvolved at best and skeptical at worst. Some, however, saw a real advantage for on-campus students to have the syllabus online, and 
others thought the academic forum was helpful in facilitating discussion through case studies or other activities. A core faculty member with expertise in both technology and online pedagogy helped faculty put those components of their on-campus courses online. Following the failure of our first effort to engage faculty, we tried two strategies - routinely presenting new developments in our online program during faculty meetings, and preparing our administrative, secretarial, and computer staff to provide support services to faculty. In general, the updates provided at faculty meetings were "background noise." Many faculty remained rather detached, though there was a slight shift in perceptions and in our academic culture as online education became a common topic. More faculty sought to use the online course templates for selected parts of their courses for on-campus students. Those who taught the basic science courses decided to post their lectures online using RealPlayer so students could revisit the class at home.

As our PFT students continued their studies, additional faculty were assigned to teach courses online to this cohort. These faculty members became fully engaged in online coursework when they had to "do it." Staff support and consultations with our early adopter or "champion" faculty, were crucial. Staff provided several essential services: entering the syllabus and related data into the course templates, making hyperlinks with online web sources and online journals, compressing and posting lectures using RealPlayer, entering quizzes, tests, and course evaluations, and in general, giving courses an aesthetically pleasing look. Faculty champions consulted on pedagogy and approaches to teaching online. Administration provided release time for course preparation. This level of support allowed faculty to focus on student learning rather than the delivery system. Once faculty had successful experience in teaching online, they began to explore new approaches, discussed pedagogical approaches and student progress with colleagues, and became comfortable with the technology. The new medium was not the message - but it came to be seen as a powerful way to teach. It was viewed as a means of making learning more accessible, effective and enjoyable for all of our students. The innovations developed for our PFT students are now integrated into our on-campus classes. Faculty view web-based course material, forums, online learning tools, and other electronic educational experiences as necessary to allow students to develop the skills needed to succeed in a digital world.

\section{COMPLEMENTARY STRATEGIES}

In addition to the online university we use three other strategies to promote learning, belonging, shared values, and collegiality. Prior to the start of coursework, students spend four days together with faculty in orientation. The first session is a 2-day orientation for all students held at the North Carolina Museum of Life and Science in Durham. Families are invited for part of the activities. Students engage in team building activities, take learning style and the Briggs tests, have hands-on computer work and sessions on diversity and time management. The second orientation is held at the student's official university - Duke or East Carolina University, where students engage in additional learning activities with faculty, tour the campus and complete tasks such as obtaining their student nurse practitioner badges. We also work with our partner professional associations to match each student with a "mentor" who lives in the student's region. Mentors serve as professional resources and role models for their protégées. Finally, based on recommendations from students and faculty, we now bring students to campus once a semester to have in-person sessions with their faculty. Most often, these sessions are held at the beginning of the semester and focus on discussing the course and helping students and faculty get to know one another. Campus sessions are usually held on Saturdays and are always scheduled at least 6 weeks in advance to accommodate students who need to change work schedules. 
Have we developed an opportunity for access to an online "academic community"? We think so. And contrary to some opinions, we think it is important - at least for those learning to assume new roles as primary care providers. Levine, in the New York Times OP-ED page noted that older part time students said they wanted a relationship with their college similar to the one they had with their utility company or supermarket - "their emphasis was on convenience, service, quality and affordability"[4]. Our experience has been somewhat different. We do not debate the value of convenience, service, quality or affordability. We continue to upgrade our online university by adding new resources and services, and faculty strive to present high quality coursework. Students can and do access their courses at all hours of the day and night as many times as they chose. Our online university increases the affordability of education since students can continue to work, stay in their community and study part time. But our students and the faculty they work with have additional needs and goals. They seek long-term collegial relationships and a professional sense of identity and shared values.

Access has many meanings. Our mission is to provide educational opportunities for rural students and access to health care for residents in rural areas of our state. The web-based format using an asynchronous learning mode provides opportunities to bring not just coursework, but the resources of our university to rural students. Since we began our journey with asynchronous online learning, software course packages have become available commercially. For those engaged in delivering a degree program in the health professions, we recommend viewing such software as part of a larger system that brings the university and its resources to students. Technology should not drive pedagogy. We also recommend a thoughtful approach to preparing students and faculty for online educational delivery systems. Such preparation ensures that the focus of the educational experience is learning, the development of collegial relationships and professional socialization.

\section{REFERENCES}

1. East Carolina Health Services Research and Development. Rates calculated by Alton Rucker and John Spencer, 1995.

2. East Carolina Health Services Research and Development and the Cecil G. Sheps Center for Health Services Research, the University of North Carolina at Chapel Hill. Calculated from data published in the 1994 Directory of Physicians in Eastern North Carolina.

3. North Carolina Health Professions Databook. 1995.

4. Levine, Arthur. "The Soul of the University", New York Times OP-ED, March 13, 2000.

\section{ABOUT THE AUTHORS}

Mary T. Champagne is Dean of the Duke University School of Nursing. PhD, University of Texas (Austin), 1981; MSN, University of Texas (Austin), 1975; BSN, San Jose State College, 1968. She is the project director for the Duke-East Carolina University Partnerships for Training Program. Address: Box 3322, Duke University Medical Center, Durham, NC 27710; telephone: 919-684-3786 Ext. 225; email: champ001@mc.duke.edu.

Donna Hewitt, RN, MN, professor emeritus, was a faculty member and Director of Special Projects in the Duke University School of Nursing. Prior to her retirement in 2001, Professor Hewitt oversaw all computer hardware, software applications, and technology personnel within the School of Nursing. She led the School in building capacity for online learning. 
Nancy M. Short is Assistant Dean for Special Projects at the Duke University School of Nursing. She has recently administered a cutting edge, web-based curriculum for nurse practitioners, physician assistants and certified nurse midwives at the Duke University School of Nursing and East Carolina University. She has published numerous articles on distance education and also workplace issues for nurses. She holds the BSN and MBA degrees from Duke University and is a candidate for the DrPH degree at the UNC-CH School of Public Health.

Susan Pietrangelo-Brown, MSN, RN, FNP, formerly an Assistant Clinical Professor with the Duke University School of Nursing, served as the Curriculum Coordinator and Lead Online Instructor for the Duke-ECU Partnerships for Training Program. She was responsible for developing and implementing key courses within the curriculum, including the interdisciplinary Physical Assessment and Diagnostic Reasoning course, as well as the five clinical courses for the FNP track. Susan also taught in the Health Promotion and Pharmacology courses. Susan created the Computer Skills Assessment Tool, used to evaluate students' self-reported computer skills. In addition, she created, implemented and maintained the Faculty Instructional Technology Resource, available to both Duke and ECU faculty members, to assist in the transition to teaching asynchronously. Susan also served as primary faculty in the School's campus-based Family Nurse Practitioner Program, and held an adjunct appointment in the East Carolina University School of Nursing. Susan Brown may be contacted via email at msmbrown@nc.rr.com.

Susan Epstein, MPA, is Chief of the Division of Community Health at Duke University. MPA, University of New Hampshire, 1974; BA, Connecticut College, 1970. Address: Division of Community Health, Box 2914, Duke University Medical Center, Durham, NC 27710; Telephone: 919-681-3187; Fax: 919-681-3371; email: epste003@mc.duke.edu. Susan Epstein served as the project director for the Duke-East Carolina University Partnerships for Training Program during the two year planning period, building the relationships and systems to support the implementation of the program. She currently serves at Chief of the Duke Division of Community Health, a joint Division between the Department of Community and Family Medicine and the School of Nursing, creating inter-disciplinary, multi-agency community based programs for populations who face barriers to primary care. She previously served as Acting Director of the New Hampshire Division of Public Health Services, Deputy Director of the NH Medicaid Program, Director of Plan Implementation for the statewide Health Systems Agency, and Executive Director of Lamprey Health Care, a section 330 Community Health Center. She held policy positions in New York City and the Commonwealth of Massachusetts.

Margaret T. Bowers, BSN, MSN, CS, FNP is an Assistant Clinical Professor in the Duke University School of Nursing and a Nurse Practitioner in the Heart Failure Disease Management Program at Duke University Medical Center. Post Master's certificate, Duke University 1998; MSN Duke University, 1990, BSN, Binghamton University, 1981. Margaret Bowers has been a primary faculty member in the Duke-East Carolina University Partnership for Training Program. She has coordinated and taught in a variety of classes including: Pharmacology, Physical Assessment and Managing Acute and Chronic Health Problems. Her experience in distance education is clinically focused and she has experience with a variety of techniques in the delivery of online coursework. Margaret has clinical experience as a Critical Care Clinical Nurse Specialist for an air and ground transport program at Duke University Medical Center. She has a variety of experience in both inpatient and outpatient cardiology and is the primary faculty in the Adult Nurse Practitioner Cardiovascular Program at Duke University School of Nursing. Margaret maintains an active clinical practice as a Nurse Practitioner managing patients in a Heart Failure clinic. Address: Duke University, School of Nursing, Box 3322 Medical Center, 
JALN Volume 6, Issue 2 - August 2002, Special Issue on Nursing

Durham, NC 27710; telephone 919-681-3244; email: bower005@mc.duke.edu; home page:

http://duson.mc.duke.edu/index.nsf/b5fd44e9908afc2e8525662500544199/e2833bb7f64c203285

2568dd005e4e79? OpenDocument. 